Corrigendum

\title{
Corrigendum to "Targeting V-ATPase Isoform Restores Cisplatin Activity in Resistant Ovarian Cancer: Inhibition of Autophagy, Endosome Function, and ERK/MEK Pathway"
}

\author{
Arpita Kulshrestha $\mathbb{D}^{\mathbb{D}},{ }^{1}$ Gajendra K. Katara, ${ }^{1}$ Safaa A. Ibrahim, ${ }^{1,2}$ Valerie Riehl, ${ }^{1}$ \\ Manoranjan Sahoo, ${ }^{1}$ James Dolan, ${ }^{3}$ Kyle W. Meinke, ${ }^{3}$ Michael R. Pins, ${ }^{4}$ \\ and Kenneth D. Beaman $\mathbb{D}^{1}$ \\ ${ }^{1}$ Department of Microbiology and Immunology, Rosalind Franklin University of Medicine and Science, North Chicago, IL, USA \\ ${ }^{2}$ Department of Microbiology and Immunology, Faculty of Pharmacy, Cairo University, Giza, Egypt \\ ${ }^{3}$ Department of Obstetrics \& Gynecology, Advocate Lutheran General Hospital, Park Ridge, IL, USA \\ ${ }^{4}$ Department of Pathology, Advocate Lutheran General Hospital, Park Ridge, IL, USA
}

Correspondence should be addressed to Kenneth D. Beaman; kenneth.beaman@rosalindfranklin.edu

Received 3 February 2021; Accepted 3 February 2021; Published 2 June 2021

Copyright ( 2021 Arpita Kulshrestha et al. This is an open access article distributed under the Creative Commons Attribution License, which permits unrestricted use, distribution, and reproduction in any medium, provided the original work is properly cited.

In the article titled "Targeting V-ATPase Isoform Restores Cisplatin Activity in Resistant Ovarian Cancer: Inhibition of Autophagy, Endosome Function, and ERK/MEK Pathway" [1], there was an error in Figure 5(a). Specifically, the shV0a2-cis- $\mathrm{R}+$ Cisplatin panel is a low magnification image of the sh-scr-cis- $\mathrm{R}+$ Cisplatin panel. The authors state that this occurred inadvertently during revision of the article and have provided the correct image for the sh-V0a2-cis- $\mathrm{R}+$ Cisplatin panel. This correction is at the request of the authors, and they confirmed that this does not affect the results and conclusions of the article. The corrected Figure 5(a) is as follows. 

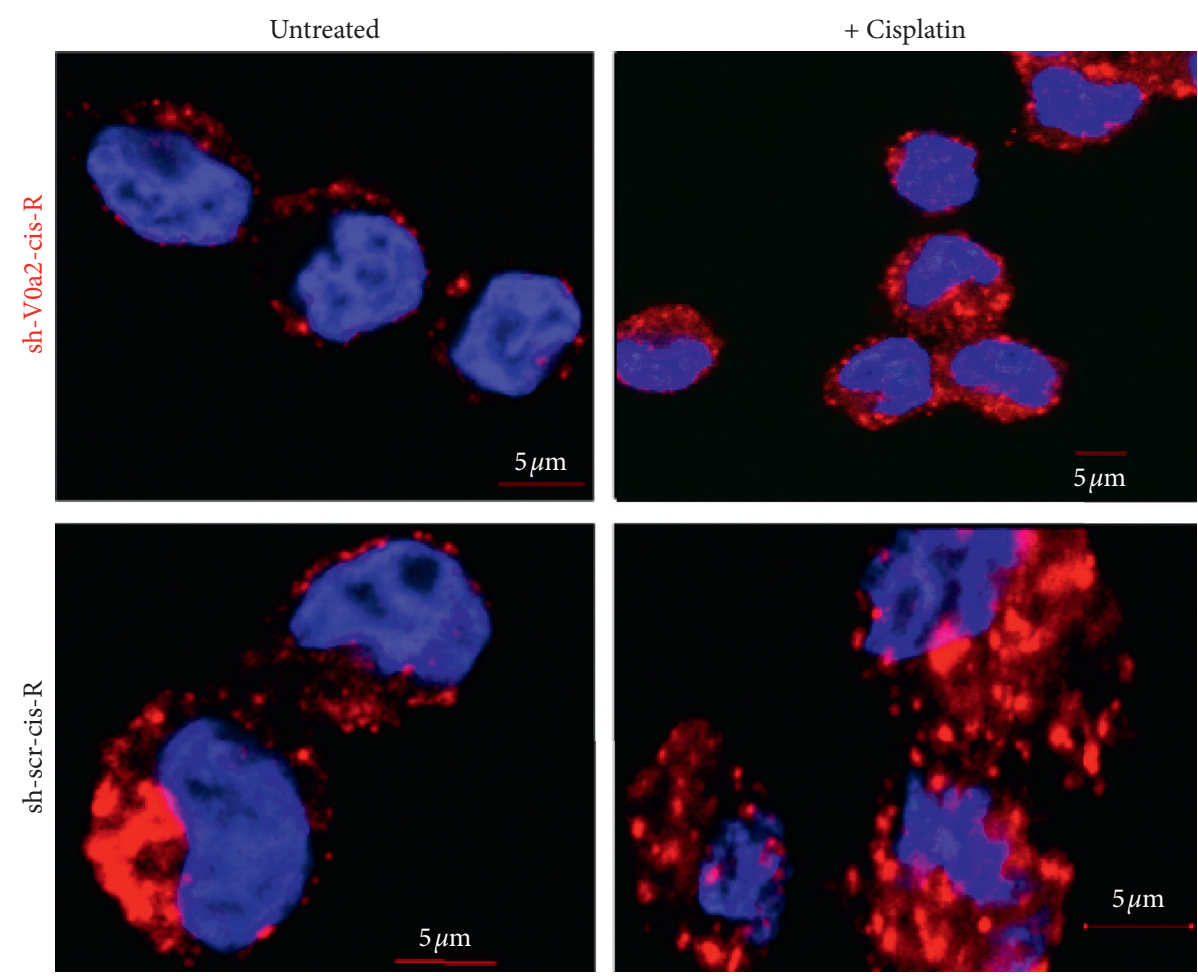

FIGURE 5: Cisplatin induces protective autophagy in V-ATPase-V0a2 inhibited resistant ovarian cancer cells with a concomitant block in autophagy flux leading to drug sensitization. V-ATPase-V0a2 inhibited cisplatin resistant ovarian cancer cells (sh-V0a2-cisR) were treated with cisplatin $(20 \mu \mathrm{g} / \mathrm{ml}, 48 \mathrm{~h})$. (a) Confocal microscopy analysis of the subcellular distribution of LC3B in V ATPase-V0a2 inhibited (sh$\mathrm{V0a} 2$-cisR) and control (sh-scr-cis-R) cisplatin resistant ovarian cancer cells, with and without cisplatin treatment. LC3B staining (red), nucleus is stained with DAPI (blue). The fluorescence images were acquired using an Olympus FluoView confocal microscope.

\section{References}

[1] A. Kulshrestha, G. K. Katara, S. A. Ibrahim et al., "Targeting V-ATPase Isoform Restores Cisplatin Activity in Resistant Ovarian Cancer: Inhibition of Autophagy, Endosome Function, and ERK/MEK Pathway," Journal of Oncology, vol. 2019, Article ID 2343876, 15 pages, 2019. 